\title{
Second order iterative functional equations related to a competition equation
}

\section{Peter Kahlig and Janusz Matkowski}

Dedicated to Professor János Aczél on the occasion of his 90th birthday

Abstract. The functional equation related to competition ([2])

$$
f\left(\frac{x+y}{1-x y}\right)=\frac{f(x)+f(y)}{1+f(x) f(y)}, \quad x, y \in \mathbb{R}, x y \neq 1,
$$

for $y=c x$ with a fixed $c>0$, leads to the equation

$$
f\left(\frac{(1+c) x}{1-c x^{2}}\right)=\frac{f(x)+f(c x)}{1+f(x) f(c x)}, \quad x \in \mathbb{R},|x|<\frac{1}{\sqrt{c}} .
$$

The case $c=1$ (a first order iterative functional equation) was treated in [3]. In this paper we consider the case $c \neq 1$ (when the equation is of the second order). We show that $a$ function $f: \mathbb{R} \rightarrow \mathbb{R}, f(0)=0$, differentiable at the point 0 satisfies this functional equation iff there is a real $p$ such that $f=\tanh \circ\left(p \tan ^{-1}\right)$ which extends the main result of [3].

Mathematics Subject Classification. Primary 39B12, 39B22.

Keywords. Functional equation, competition equation, iterative functional equation of the second order, differentiable solution, solution depending on an arbitrary function.

\section{Introduction}

The functional equation on a restricted domain (following [1] a conditional functional equation),

$$
f\left(\frac{x+y}{1-x y}\right)=\frac{f(x)+f(y)}{1+f(x) f(y)}, \quad x, y \in \mathbb{R}, x y \neq 1,
$$

the so-called competition equation, considered first in [2], was also treated in [3]. If $f$ is a solution then either $f(0)=0$, or $f(0)=-1$ or $f(0)=1$. It is 
shown in [2] (cf. also [3]) that, if $f$ takes value -1 or 1 , then it is a constant function (cf. also Remark 1). Therefore only the case $f(0)=0$ is interesting. The main result of [3] says that, in this case, a function $f: \mathbb{R} \rightarrow \mathbb{R}$ satisfies the equation for all real $x, y$ such that $x y<1$, iff $f=\tanh \circ \alpha \circ \tan ^{-1}$ where $\alpha: \mathbb{R} \rightarrow \mathbb{R}$ is an additive function. Thus, if $f$ is measurable or continuous at least at one point, then there is a $p \in \mathbb{R}$ such that $f=g_{p}$ where

$$
g_{p}=\tanh \circ\left(p \tan ^{-1}\right) .
$$

Taking $y=x$ in $(\mathrm{CE})$ we obtain

$$
f\left(\frac{2 x}{1-x^{2}}\right)=\frac{2 f(x)}{1+f(x)^{2}}, \quad x \in \mathbb{R}, x^{2} \neq 1,
$$

the diagonalization of the competition equation, which is a first order iterative functional equation (cf. $[5,6]$ ). In [2] it is proved that if a function $f: \mathbb{R} \rightarrow \mathbb{R}$, such that $f(0)=0$, satisfies this equation for all $x \in(-1,1)$, and is twice differentiable at the point 0 , then $f=g_{p}$ for some real $p$.

In [4] the following stronger result is presented. A function $f: \mathbb{R} \rightarrow \mathbb{R}$, $f(0)=0$, differentiable at the point 0 , satisfies this functional equation iff there is a real $p$ such that $f=g_{p}$. Moreover $g_{p}^{\prime}(0)=p$ (Theorem 1 ). Applying the theory of iterative functional equations $([5,6]$, cf. also $[7,8])$ it is also shown that in this result the assumption of the differentiability of the solution $f$ at the point 0 cannot be replaced by the continuity of $f$ at 0 .

In the present paper we consider a generalization of the above diagonalization problem. Namely, in Sect. 2, for a fixed $c>0$, restricting the competition equation to the straight line $y=c x$, we obtain the iterative functional equation

$$
f\left(\frac{(1+c) x}{1-c x^{2}}\right)=\frac{f(x)+f(c x)}{1+f(x) f(c x)}, \quad x \in \mathbb{R},|x|<\frac{1}{\sqrt{c}},
$$

which is of the second order if $c \neq 1$. (For the definition of the $n$th order of an iterative functional equation see [5], chapter XII, and [6] pp. 237-239.) Our main result says that a function $f: \mathbb{R} \rightarrow \mathbb{R}$, differentiable at the point 0 and such that $f(0)=0$, satisfies this equation iff there is a $p \in \mathbb{R}$ such that $f=g_{p}$, where $g_{p}=\tanh \circ\left(p \tan ^{-1}\right)$.

In Sect. 3 we discuss the case when $c \leq 0$.

In Sect. 4, recalling the motivation coming from a meteorological phenomenon (hail suppression by competition of small particles via cloud seeding), we discuss the mutual relation between $(\mathrm{CE})$ and a Riccati differential equation.

\section{Main result}

Let us fix $c>0$. In this section we consider the competition equation restricted to the straight line $\{(x, c x): x \in \mathbb{R}\}$. 
Theorem 1. Let $c>0$ be arbitrarily fixed. Suppose that $f: \mathbb{R} \rightarrow \mathbb{R}$ is differentiable at the point 0 and $f(0)=0$. If $f$ satisfies the functional equation

$$
f\left(\frac{(1+c) x}{1-c x^{2}}\right)=\frac{f(x)+f(c x)}{1+f(x) f(c x)}, \quad|x|<\frac{1}{\sqrt{c}},
$$

then there is a $p \in \mathbb{R}$ such that $f=g_{p}$, where $g_{p}=\tanh \circ\left(p \tan ^{-1}\right)$.

Proof. It is easy to verify that if $f: \mathbb{R} \rightarrow \mathbb{R}$ is differentiable at the point $0, f(0)=0$, and $f$ satisfies equation (1), then the function $\varphi: \mathbb{R} \rightarrow \mathbb{R}$ defined by

$$
\varphi(x):=\left\{\begin{array}{cc}
\frac{f(x)}{x} & \text { for } x \neq 0 \\
p & \text { for } x=0
\end{array},\right.
$$

where

$$
p:=f^{\prime}(0),
$$

is continuous at 0 and satisfies the functional equation

$$
\frac{(1+c)}{1-c x^{2}} \varphi\left(\frac{(1+c) x}{1-c x^{2}}\right)=\frac{\varphi(x)+c \varphi(c x)}{1+c x^{2} \varphi(x) \varphi(c x)}, \quad|x|<\frac{1}{\sqrt{c}} .
$$

Suppose that the functions $\varphi_{1}: \mathbb{R} \rightarrow \mathbb{R}$ and $\varphi_{2}: \mathbb{R} \rightarrow \mathbb{R}$ satisfy this equation, are continuous at the point 0 and

$$
\varphi_{1}(0)=\varphi_{2}(0)=p .
$$

Put

$$
I_{c}:=\left(-\frac{1}{\sqrt{c}}, \frac{1}{\sqrt{c}}\right) .
$$

The function $h: I_{c} \rightarrow \mathbb{R}$ defined by

$$
h(x)=\frac{(1+c) x}{1-c x^{2}}, \quad x \in I_{c},
$$

is odd, continuous, strictly increasing, convex in $(0, \infty)$, concave in $(-\infty, 0)$, maps $I_{c}$ onto $\mathbb{R}$ and

$$
h^{\prime}(0)=1+c .
$$

It follows that its inverse $\beta:=h^{-1}$ is odd, continuous, increasing, convex in $(-\infty, 0)$, concave in $(0, \infty)$, maps $\mathbb{R}$ onto $I_{c}$ and

$$
\beta^{\prime}(0)=\frac{1}{1+c}<1
$$

Of course, the function $\gamma:=c \beta$ has similar properties. Moreover

$$
\begin{aligned}
& \gamma^{\prime}(0)=\frac{c}{1+c}<1, \\
& 0<\frac{\beta(t)}{t}<1, \quad 0<\frac{\gamma(t)}{t}<1, \quad t \in \mathbb{R}, t \neq 0,
\end{aligned}
$$


and, consequently, for every $t \in \mathbb{R}$, the sequences $\left(\beta^{n}\right)_{n \in \mathbb{N}}$ and $\left(\gamma^{n}\right)_{n \in \mathbb{N}}$ of iterates of the functions $\beta$ and $\gamma$ converge uniformly to 0 on compact subsets of $\mathbb{R}$.

With the above solutions $\varphi_{1}, \varphi_{2}$ of equation (2), we have

$$
\frac{(1+c)}{1-c x^{2}} \varphi_{j}\left(\frac{(1+c) x}{1-c x^{2}}\right)=\frac{\varphi_{j}(x)+c \varphi_{j}(c x)}{1+c x^{2} \varphi_{j}(x) \varphi_{j}(c x)}, \quad x \in I_{c}, \quad j=1,2 .
$$

Hence, putting

$$
\psi=\varphi_{1}-\varphi_{2},
$$

and subtracting the respective sides of these equalities for $j=1$ and $j=2$, we get, for all $x \in I_{c}$,

$$
\begin{aligned}
\varphi_{1} & \left(\frac{(1+c) x}{1-c x^{2}}\right)-\varphi_{2}\left(\frac{(1+c) x}{1-c x^{2}}\right)=\psi\left(\frac{(1+c) x}{1-c x^{2}}\right) \\
& =\frac{1-c x^{2}}{1+c} \frac{\psi(x)\left[1-c^{2} x^{2} \varphi_{1}(c x) \varphi_{2}(c x)\right]+c \psi(c x)\left[1-x^{2} \varphi_{1}(x) \varphi_{2}(x)\right]}{\left[1+c x^{2} \varphi_{1}(x) \varphi_{1}(c x)\right]\left[1+c x^{2} \varphi_{2}(x) \varphi_{2}(c x)\right]} .
\end{aligned}
$$

Since $\varphi_{1}$ and $\varphi_{2}$ are continuous at 0 and $\varphi_{1}(0)=\varphi_{2}(0)$, there is $\delta>0$ such that

$$
\begin{gathered}
1-c^{2} x^{2} \varphi_{1}(c x) \varphi_{2}(c x)>0 ; \quad 1-x^{2} \varphi_{1}(x) \varphi_{2}(x)>0 ; \\
1+c x^{2} \varphi_{j}(x) \varphi_{j}(c x)>0 \quad(j=1,2),
\end{gathered}
$$

and $1-c x^{2}>0$ for all $x \in(-\delta, \delta)$. Hence, setting

$$
\begin{aligned}
& k(x):=\frac{1-c x^{2}}{1+c} \frac{1-c^{2} x^{2} \varphi_{1}(c x) \varphi_{2}(c x)}{\left[1+c x^{2} \varphi_{1}(x) \varphi_{1}(c x)\right]\left[1+c x^{2} \varphi_{2}(x) \varphi_{2}(c x)\right]}, \\
& m(x):=\frac{1-c x^{2}}{1+c} \frac{c\left[1-x^{2} \varphi_{1}(x) \varphi_{2}(x)\right]}{\left[1+c x^{2} \varphi_{1}(x) \varphi_{1}(c x)\right]\left[1+c x^{2} \varphi_{2}(x) \varphi_{2}(c x)\right]},
\end{aligned}
$$

we have

$$
k(x)>0, \quad m(x)>0, \quad x \in(-\delta, \delta),
$$

and

$$
\left|\psi\left(\frac{(1+c) x}{1-c x^{2}}\right)\right| \leq k(x)|\psi(x)|+m(x)|\psi(c x)|, \quad x \in(-\delta, \delta) .
$$

Moreover, for $x \in(-\delta, \delta)$, we have

$$
\begin{aligned}
k(x)+m(x) & \leq \frac{1}{1+c} \frac{1-c^{2} x^{2} \varphi_{1}(c x) \varphi_{2}(c x)+c\left[1-x^{2} \varphi_{1}(x) \varphi_{2}(x)\right]}{\left[1+c x^{2} \varphi_{1}(x) \varphi_{1}(c x)\right]\left[1+c x^{2} \varphi_{2}(x) \varphi_{2}(c x)\right]} \\
& \leq \frac{1}{1+c} \frac{1+c}{\left[1+c x^{2} \varphi_{1}(x) \varphi_{1}(c x)\right]\left[1+c x^{2} \varphi_{2}(x) \varphi_{2}(c x)\right]} \leq 1 .
\end{aligned}
$$

Setting $x=\beta(t)$ in inequality (3) and considering the definition of $\gamma$, we get

$$
|\psi(t)| \leq \kappa(t)|\psi(\beta(t))|+\mu(t)|\psi(\gamma(t))|, \quad t \in I:=\beta^{-1}(-\delta, \delta),
$$


where $\kappa(t)=k(\beta(t)), \mu(t)=m(\beta(t))$, hence

$$
\kappa(t)+\mu(t) \leq 1, \quad t \in I,
$$

and, of course, $I=\beta^{-1}(-\delta, \delta)$ is an open interval such that $0 \in I$.

Define $\sigma: \mathbb{R} \rightarrow \mathbb{R}$ by

$$
\sigma(t):=\left\{\begin{array}{ll}
\max (\beta(t), \gamma(t)) & \text { for } t \geq 0 \\
\min (\beta(t), \gamma(t)) & \text { for } t<0
\end{array} .\right.
$$

Of course, $\sigma$ is continuous, strictly increasing and

$$
0<\frac{\sigma(t)}{t}<1, \quad t \in \mathbb{R}, t \neq 0 .
$$

From inequalities (4) and (5) we obtain, for all $t \in I$,

$$
\begin{aligned}
|\psi(t)| & \leq \kappa(t) \sup |\psi(\beta(I))|+\mu(t) \sup |\psi(\gamma(I))| \\
& \leq(\kappa(t)+\mu(t)) \sup |\psi(\sigma(I))|=\sup |\psi(\sigma(I))|,
\end{aligned}
$$

whence

$$
\sup |\psi(I)| \leq \sup |\psi(\sigma(I))|
$$

Hence, by induction,

$$
\sup |\psi(I)| \leq \sup \left|\psi\left(\sigma^{n}(I)\right)\right|, \quad n \in \mathbb{N},
$$

where $\sigma^{n}$ denotes the $n$-th iterate of $\sigma$. Since the decreasing sequence of intervals $\left(\sigma^{n}(I)\right)_{n \in \mathbb{N}}$ tends to $\{0\}$, this inequality, the continuity of $\psi$ at 0 and $\psi(0)=0$ imply that $\sup |\psi(I)|=0$. Hence, from the definition of $\psi$ we get $\varphi_{1}(x)=\varphi_{2}(x)$ for all $x \in \dot{I}$. Now, according to the theory of iterative functional equations (cf. [5], p. 68, Lemma 3.1) we conclude that $\varphi_{1}=\varphi_{2}$. Taking into account the definition of the function $\varphi$, we have proved that, for any $p \in \mathbb{R}$, there exists at most one solution $f$ of equation (1) that is differentiable at 0 and such that $f^{\prime}(0)=p$. Since, in view of Lemma 1 , the function $f=g_{p}$ satisfies equation (1), is differentiable at 0 and $f^{\prime}(0)=p$, the proof is complete.

To justify the assumption $f(0)=0$ consider the following

Remark 1. Suppose that $f: \mathbb{R} \rightarrow \mathbb{R}$ is a solution of the competition equation (CE). Setting $x=y=0$ in (CE) we obtain $f(0)=\frac{2 f(0)}{1+[f(0)]^{2}}$ whence either $f(0)=0$ or $f(0)=1$ or $f(0)=-1$.

If $f(0)=1$ then, setting $y=0$ in (CE), we get $f(x)=1$ for all $x \in \mathbb{R}$.

If $f(0)=-1$ then, setting $y=0$ in $(\mathrm{CE})$, we get $f(x)=-1$ for all $x \in \mathbb{R}$. 


\section{Discussion of the case when $c$ is nonpositive}

In this section we consider the functions $f: \mathbb{R} \rightarrow \mathbb{R}, f(0)=0$, satisfying the competition equation (CE) restricted to the straight line $\{(x, c x): x \in \mathbb{R}\}$, i.e. the equation

$$
f\left(\frac{(1+c) x}{1-c x^{2}}\right)=\frac{f(x)+f(c x)}{1+f(x) f(c x)},
$$

where $c \leq 0$.

Remark 2. For $c=0$ this equation reduces to the identity $f(x)=f(x)$ for all $x \in \mathbb{R}$, so every function $f: \mathbb{R} \rightarrow \mathbb{R}, f(0)=0$, is a solution.

For $c=-1$ this equation reduces to the equality $0=f(x)+f(-x)$, so $f: \mathbb{R} \rightarrow \mathbb{R}$ is a solution iff it is an odd function.

Remark 3. The counterpart of Theorem 1 holds true in the case $c<0$ and $c \neq-1$.

Assume, for instance that $-1<c<0$; setting $y=-c x$ in the competition equation $(\mathrm{CE})$ we get

$$
f\left(\frac{(1-c) x}{1+c x^{2}}\right)=\frac{f(x)+f(-c x)}{1+f(x) f(-c x)}, \quad x \in \mathbb{R},
$$

whence, calculating $f(x)$, we get the functional equation

$$
f(x)=\frac{f\left(\frac{(1-c) x}{1+c x^{2}}\right)-f(-c x)}{1-f(-c x) f\left(\frac{(1-c) x}{1+c x^{2}}\right)}, \quad x \in \mathbb{R} .
$$

If $f: \mathbb{R} \rightarrow \mathbb{R}$ is differentiable at the point $0, f(0)=0$, and $f$ satisfies this equation, then the function $\varphi: \mathbb{R} \rightarrow \mathbb{R}$ defined by

$$
\varphi(x):=\left\{\begin{array}{ccc}
\frac{f(x)}{x} & \text { for } & x \neq 0 \\
p & \text { for } & x=0
\end{array},\right.
$$

where

$$
p:=f^{\prime}(0),
$$

is continuous at 0 and satisfies the functional equation

$$
\varphi(x)=\frac{\frac{1-c}{1+c x^{2}} \varphi\left(\frac{(1-c) x}{1+c x^{2}}\right)+c \varphi(-c x)}{1+\frac{c(1-c) x^{2}}{1+c x^{2}} \varphi(-c x) \varphi\left(\frac{(1-c) x}{1+c x^{2}}\right)}, \quad x \in \mathbb{R},
$$

whence, setting

$$
h(x):=\frac{(1-c) x}{1+c x^{2}}, \quad x \in \mathbb{R},
$$


we can write this equation in the form

$$
\varphi(x)=\frac{(1-c) \varphi(h(x))+c\left(1+c x^{2}\right) \varphi(-c x)}{1+c x^{2}+c(1-c) x^{2} \varphi(-c x) \varphi(h(x))}, \quad x \in \mathbb{R} .
$$

Suppose that the functions $\varphi_{1}: \mathbb{R} \rightarrow \mathbb{R}$ and $\varphi_{2}: \mathbb{R} \rightarrow \mathbb{R}$ satisfy this equation, are continuous at the point 0 and

$$
\varphi_{1}(0)=\varphi_{2}(0)=p .
$$

Hence we have

$$
\varphi_{j}(x)=\frac{(1-c) \varphi_{j}(h(x))+c\left(1+c x^{2}\right) \varphi_{j}(-c x)}{1+c x^{2}+c(1-c) x^{2} \varphi_{j}(-c x) \varphi_{j}(h(x))}, \quad x \in \mathbb{R} ; j=1,2 .
$$

Setting

$$
\begin{aligned}
& \psi:=\varphi_{1}-\varphi_{2}, \quad y_{1}=y_{1}(x)=\varphi_{1}(h(x)), \quad y_{2}=y_{2}(x)=\varphi_{1}(-c x), \\
& z_{1}=z_{1}(x)=\varphi_{2}(h(x)), \quad z_{2}=z_{2}(x)=\varphi_{2}(-c x), \quad(x \in \mathbb{R}),
\end{aligned}
$$

and subtracting the respective sides of these equalities for $j=1$ and $j=2$, performing simple calculations, we get

$\psi(x)$

$$
=\frac{(1-c)\left[1+c x^{2}-c^{2} x^{2}\left(1+c x^{2}\right) y_{2} z_{2}\right] \psi(h(x))+\left[c\left(1+c x^{2}\right)-c(1-c)^{2} x^{2} y_{1} z_{1}\right] \psi(-c x)}{\left[1+c x^{2}+c(1-c) x^{2} y_{1} y_{2}\right]\left[1+c x^{2}+c(1-c) x^{2} z_{1} z_{2}\right]}
$$

for $x \in \mathbb{R}$, whence

$$
\psi(x)=\lambda(x) \psi(h(x))+\mu(x) \psi(-c x), \quad x \in \mathbb{R},
$$

where

$$
\begin{array}{ll}
\lambda(x):=\frac{(1-c)\left[1+c x^{2}-c^{2} x^{2}\left(1+c x^{2}\right) y_{2} z_{2}\right]}{\left[1+c x^{2}+c(1-c) x^{2} y_{1} y_{2}\right]\left[1+c x^{2}+c(1-c) x^{2} z_{1} z_{2}\right]}, & x \in \mathbb{R}, \\
\mu(x):=\frac{c\left(1+c x^{2}\right)-c(1-c)^{2} x^{2} y_{1} z_{1}}{\left[1+c x^{2}+c(1-c) x^{2} y_{1} y_{2}\right]\left[1+c x^{2}+c(1-c) x^{2} z_{1} z_{2}\right]}, & x \in \mathbb{R} .
\end{array}
$$

Since the functions $y_{1}, y_{2}, z_{1}, z_{2}$ are continuous at $x=0$ and

$$
y_{1}(0)=y_{2}(0)=z_{1}(0)=z_{2}(0)=p,
$$

it is easy to check that there is a real number $\delta>0$ such that

$$
\lambda(x) \geq 0, \quad \mu(x) \geq 0, \quad \lambda(x)+\mu(x) \leq 1, \quad x \in(-\delta, \delta) .
$$

Hence, making use of (6), we obtain

$$
|\psi(x)| \leq \lambda(x)|\psi(h(x))|+\mu(x)|\psi(-c x)|, \quad x \in(-\delta, \delta) .
$$

Now we can argue similarly as in the proof of Theorem 1. 


\section{Motivation and remarks on a Riccati-type differential equation and the competition functional equation (CE)}

In [2] it was shown that a model of a meteorological phenomenon in cloud physics, interpreted as competition and described with the aid of a Riccati differential equation, can be fully characterized by the functional equation (CE) that does not involve any derivative. The functional equation (CE) reflects symmetry properties of the corresponding model differential equation (and of its solution).

Remark 4. (Differential equation and initial condition)

Some physical models present themselves in this way,

$$
\frac{d F(X)}{d X}=\frac{1-b[F(X)]^{2}}{1+a X^{2}}, \quad X \in \mathbb{R},
$$

with two arbitrary parameters $a, b>0$, fixed. (In [2] there is $a=b=1$.) This may be reduced to a one-parameter model: (7) can be written in the form

$$
\frac{d\left[\sqrt{b} F\left(\frac{1}{\sqrt{a}}(\sqrt{a} X)\right)\right]}{\sqrt{b}\left(\frac{1}{\sqrt{a}} d(\sqrt{a} X)\right)}=\frac{1-\left[\sqrt{b} F\left(\frac{1}{\sqrt{a}}(\sqrt{a} X)\right)\right]^{2}}{1+(\sqrt{a} X)^{2}},
$$

whence

$$
\frac{d\left[\sqrt{b} F\left(\frac{1}{\sqrt{a}}(\sqrt{a} X)\right)\right]}{d(\sqrt{a} X)}=\frac{\sqrt{b}}{\sqrt{a}} \frac{1-\left[\sqrt{b} F\left(\frac{1}{\sqrt{a}}(\sqrt{a} X)\right)\right]^{2}}{1+(\sqrt{a} X)^{2}} .
$$

Therefore, setting

$$
f(x)=\sqrt{b} F\left(\frac{1}{\sqrt{a}} x\right), \quad x=\sqrt{a} X,
$$

we can write this equation in the form

$$
\frac{d f(x)}{d x}=\frac{\sqrt{b}}{\sqrt{a}} \frac{1-f(x)^{2}}{1+x^{2}} .
$$

Hence, if $F(0)=0=f(0)$ then $p=f^{\prime}(0)=\frac{\sqrt{b}}{\sqrt{a}}$ and, consequently, we can write this equation in the form

$$
\frac{d f(x)}{d x}=p \frac{1-f(x)^{2}}{1+x^{2}}, \quad x \in \mathbb{R},
$$

a nonlinear first-order differential equation of Riccati-type. Here we have only one parameter, namely $p>0$, fixed. (In [2], as $a=b=1$, we have $f=F$ and $p=1$.) 
With initial condition $f\left(x_{0}\right)=f_{0}$ (where $x_{0}, f_{0} \in \mathbb{R}$, fixed), the solution of equation (8) (obtainable by separation of variables) reads as

$$
\begin{aligned}
f(x) & =\tanh \left[p\left(\tan ^{-1}(x)-\tan ^{-1}\left(x_{0}\right)\right)+\tanh ^{-1}\left(f_{0}\right)\right] \\
& \equiv \frac{g_{p}(x)-g_{p}\left(x_{0}\right)+\left[1-g_{p}(x) g_{p}\left(x_{0}\right)\right] \cdot f_{0}}{1-g_{p}(x) g_{p}\left(x_{0}\right)+\left[g_{p}(x)-g_{p}\left(x_{0}\right)\right] \cdot f_{0}} \equiv \frac{f_{0}+g_{p}\left(\frac{x-x_{0}}{1+x x_{0}}\right)}{1+f_{0} g_{p}\left(\frac{x-x_{0}}{1+x x_{0}}\right)} .
\end{aligned}
$$

Check: $f\left(x_{0}\right)=f_{0}$, and we have $f(0)=\left[f_{0}+g_{p}\left(-x_{0}\right)\right] /\left[1+f_{0} g_{p}\left(-x_{0}\right)\right] \neq$ 0 (in general). It is to be noted that the last two versions of (9) do not require $\left|f_{0}\right|<1$ (fixed) but admit $f_{0} \in \mathbb{R}$ (fixed). In general, (9) is not an odd function of $x$. Only for the special initial condition $f(0)=0$ (i.e. $x_{0}=0, f_{0}=0$, called "standard" initial condition), the result (9) reduces to an odd function (called "standard" solution),

$$
f(x)=\tanh \left[p \tan ^{-1}(x)\right] \equiv g_{p}(x) .
$$

With this standard solution $g_{p}$ we can represent the general solution $f$ of the differential equation (8) via (9) as a rational (fractional linear) expression in $g_{p}$.

From $(10)$ we get $g_{-p}(x)=g_{p}(-x)=-g_{p}(x)$, implying that we may relax the condition $p>0$ (fixed) to $p \in \mathbb{R}$ (fixed) for $f: \mathbb{R} \rightarrow \mathbb{R}$. Already from (8) we see that changing the sign of $p$ is equivalent to changing the sign of $f$ [and $f_{0}$ in $\left.(9)\right]$.

Remark 5. If (in a certain physical situation) a slightly more general fourparameter model appears adequate or desirable (e.g. for easy interpretation),

$$
\frac{d G}{d X}=\frac{C-B G(X)^{2}}{D+A X^{2}}, \quad X \in \mathbb{R},
$$

with parameters $A, B, C, D \in \mathbb{R}$ (fixed) and $C D \neq 0$, it can be reduced immediately to the two-parameter model (7) by writing

$$
\frac{D}{C} \frac{d G}{d X}=\frac{1-\frac{B}{C} G(X)^{2}}{1+\frac{A}{D} X^{2}} \equiv \frac{1-\frac{B C}{D^{2}}\left[\frac{D}{C} G(X)\right]^{2}}{1+\frac{A}{D} X^{2}} ;
$$

abbreviating $a:=\frac{A}{D}, b:=\frac{B C}{D^{2}}, F(X):=\frac{D}{C} G(X)$, we get equation (7). [Further reduction to the one-parameter model (8) may be done as above.]

Remark 6. If a differentiable function $f: \mathbb{R} \rightarrow \mathbb{R}$ satisfies the functional equation (CE) then it satisfies the Riccati differential equation (8).

Proof. Assume that a differentiable function $f: \mathbb{R} \rightarrow \mathbb{R}$ satisfies the functional equation

$$
f\left(\frac{x+y}{1-x y}\right)=\frac{f(x)+f(y)}{1+f(x) f(y)}, \quad x, y \in \mathbb{R}, x y<1 .
$$


Differentiating both sides with respect to $x$ we get

$$
f^{\prime}\left(\frac{x+y}{1-x y}\right) \frac{1+y^{2}}{(1-x y)^{2}}=\frac{f^{\prime}(x)\left[1-f(y)^{2}\right]}{[1+f(x) f(y)]^{2}}, \quad x, y \in \mathbb{R}, x y<1,
$$

while differentiating with respect to $y$, we obtain

$$
f^{\prime}\left(\frac{x+y}{1-x y}\right) \frac{1+x^{2}}{(1-x y)^{2}}=\frac{f^{\prime}(y)\left[1-f(x)^{2}\right]}{[1+f(x) f(y)]^{2}}, \quad x, y \in \mathbb{R}, x y<1 .
$$

It follows that

$$
\frac{(1-x y)^{2}}{1+y^{2}} \frac{f^{\prime}(x)\left[1-f(y)^{2}\right]}{[1+f(x) f(y)]^{2}}=\frac{(1-x y)^{2}}{1+x^{2}} \frac{f^{\prime}(y)\left[1-f(x)^{2}\right]}{[1+f(x) f(y)]^{2}}, \quad x, y \in \mathbb{R}, x y<1,
$$

whence

$$
\frac{f^{\prime}(x)\left[1-f(y)^{2}\right]}{1+y^{2}}=\frac{f^{\prime}(y)\left[1-f(x)^{2}\right]}{1+x^{2}}, \quad x, y \in \mathbb{R}, x y<1 .
$$

Thus either there is $y \in \mathbb{R}$ such that $[f(y)]^{2} \neq 0$ and, consequently,

$$
f^{\prime}(x)=p \frac{1-[f(x)]^{2}}{1+x^{2}}, \quad x \in \mathbb{R},
$$

for some fixed $p \in \mathbb{R}$, that is, $f$ satisfies the Riccati differential equation (8), or $f=1$ or $f=-1$.

The correspondence of $(\mathrm{CE})$ and (8) is interesting: at first sight, the functional equation (CE) seems to lack the parameter $p$, whereas $p$ shows up explicitly in the differential equation (8). However, $p$ is contained implicitly in the functional equation via $p=f^{\prime}(0)$; it could be called "hidden parameter". Functional equation (CE) reflects symmetry properties of differential equation (8) (and its solution) for any value of the parameter $p$.

The equations (CE) and (8) are not equivalent (because the families of the solutions of these equations are different). Any differentiable solution of functional equation (CE) is a solution of differential equation (8) (but not conversely). One of the advantages of a functional equation is that it sometimes permits to get some solutions of a (corresponding) differential equation without (strong) differentiability conditions. (In the present paper we assume differentiability of the solution only at one point.)

Remark 7. Let $c \in \mathbb{R}, c \neq 0$, be fixed. Note that a function $\chi: \mathbb{R} \rightarrow \mathbb{R}$ satisfies the functional equation

$$
\chi\left(\frac{x+y}{1-x y}\right)=\frac{\chi(x)+\chi(y)}{1+c^{2} \chi(x) \chi(y)}, \quad x, y \in \mathbb{R}, x y \neq 1,
$$


iff $f=c \chi$ satisfies functional equation $(\mathrm{CE})$,

$$
f\left(\frac{x+y}{1-x y}\right)=\frac{f(x)+f(y)}{1+f(x) f(y)}, \quad x, y \in \mathbb{R}, x y \neq 1 .
$$

Open Access. This article is distributed under the terms of the Creative Commons Attribution License which permits any use, distribution, and reproduction in any medium, provided the original author(s) and the source are credited.

\section{References}

[1] Aczél, J., Dhombres, J.: Functional Equations in Several Variables, Encyclopedia of Mathematics and its Applications. Cambridge University Press, Cambridge (1989)

[2] Kahlig, P.: A model of competition. Appl. Math. 39, 293-303 (2012)

[3] Kahlig, P., Matkowski, J.: On a functional equation related to competition. Aequat. Math. 87, 301-308 (2014). doi:10.1007/s00010-013-0215-9

[4] Kahlig, P., Matkowski, J.: Iterative functional equations related to a competition equation. Aequat. Math. (in press). doi:10.1007/s00010-013-0248-0

[5] Kuczma, M.: Functional equations in a single variable, Monografie Matematyczne 46. PWN Warszawa (1968)

[6] Kuczma, M., Choczewski, B., Ger, R.: Iterative Functional Equations, Encyclopedia of Mathematics and its Applications. Cambridge University Press, Cambridge (1990)

[7] Matkowski, J.: On the uniqueness of differentiable solutions of a functional equation. Bull. Acad. Polon. Sci. 18, 253-255 (1970)

[8] Matkowski, J.: On the existence of differentiable solutions of a functional equation. Bull. Acad. Polon. Sci. 19, 19-22 (1971)

[9] Matkowski, J.: The uniqueness solutions of a system of functional equations in some classes of functions. Aequat. Math. 8, 233-237 (1972)

Present address

Peter Kahlig

Science Pool Vienna

Section of Hydrometeorology,

Altmannsdorfer Str. 21/5/2,

1120 Vienna, Austria

e-mail: peter.kahlig@gmx.at

Present address

Janusz Matkowski

Faculty of Mathematics

Computer Science and Econometrics

University of Zielona Góra

Szafrana 4A, 65-516 Zielona Gora

Poland

e-mail: J.Matkowski@wmie.uz.zgora.pl

Received: March 29, 2014

Revised: July 29, 2014 